\title{
Orbital-polarization terms: from a phenomenological to a first-principles description of orbital magnetism in density-functional theory
}

\author{
J. M. Morbec and K. Capelle \\ Departamento de Física e Informática \\ Instituto de Física de São Carlos \\ Universidade de São Paulo \\ Caixa Postal 369, 13560-970 São Carlos, SP \\ Brazil
}

November 8, 2018

\begin{abstract}
Phenomenological orbital-polarization (OP) terms have been repeatedly introduced in the single-particle equations of spin-density-functional theory, in order to improve the description of orbital magnetic moments in systems containing transition metal ions. Here we show that these ad hoc corrections can be interpreted as approximations to the exchangecorrelation vector potential $\mathbf{A}_{x c}$ of current-density-functional theory (CDFT). This connection provides additional information on both approaches: Phenomenological OP terms are connected to first-principles theory, leading to a rationale for their empirical success and a reassessment of their limitations and the approximations made in their derivation. Conversely, the connection of OP terms with CDFT leads to a set of simple approximations to the CDFT potential $\mathbf{A}_{x c}$, with a number of desirable features that are absent from electron-gas-based functionals.
\end{abstract}




\section{Contents}

1 Introduction

2 Orbital magnetism in many-electron systems 3

3 Phenomenological and first-principles treatment of orbital magnetism in density-functional theory

3.1 Current-density-functional theory . . . . . . . . . . 5

3.2 Orbital-polarization terms . . . . . . . . . . . . . . . .

4 Connection between the phenomenological and the first-principles approach

$4.1 \quad$ Single-particle energies . . . . . . . . . . . . . . . . . . . . . 9

4.2 Total energies . . . . . . . . . . . . . . . . . . . . 12

5 Conclusions 13

References 14 


\section{Introduction}

Magnetic phenomena arising as a consequence of the spin degrees of freedom and the antisymmetrization of the wave function are ubiquitous in quantum chemistry and physics, and are routinely treated within both density-based and wavefunction-based approaches. Magnetic phenomena arising from orbital currents, on the other hand, are not automatically accounted for by standard methods for electronic-structure calculations. The present paper is dedicated to exploring links between a phenomenological and a first-principles approach to orbital magnetism in density-functional theory (DFT). These links shed new light on both approaches, and pave the way for new applications of each.

In Sec. 2. of the present paper, we briefly recall a number of distinct ways in which orbital magnetism can occur in a many-electron system. In Sec. 3 , we describe two computational approaches to orbital magnetism. Section 3.1 is devoted to current-density functional theory (CDFT), as a first-principles method for the description of orbital magnetism in DFT $[1,2]$. Independently of CDFT, a variety of phenomenological orbital polarization (OP) terms were proposed as add-ons to the single-particle equations of spin-density-functional theory (SDFT) [3-7]. These terms are quite commonly used in band-structure calculations of magnetic solids, but are much less known in quantum chemistry. We devote Sec. 3.2 to a quick description of the motivation and form of several such add-on terms.

In Sec. 4, we show that these phenomenological terms can be related to the first-principles approach, both for single-particle energies (Sec. 4.1) and total energies (Sec. 4.2). This connection benefits both sides: Phenomenological OP terms are connected to first-principles theory, leading to a rationale for their empirical success and a reassessment of their limitations and the approximations made in their derivation. Conversely, the connection of orbital-polarization terms with CDFT leads to a set of simple approximations to the CDFT vector potential $\mathbf{A}_{x c}$ of systems containing open-shell atoms, with a number of desirable features that are absent from electron-gas-based functionals.

Section 5 contains our conclusions.

\section{Orbital magnetism in many-electron systems}

There are at least four conceptually distinct ways in which orbital magnetism can appear in a physical system. One is the presence of external magnetic fields $\mathbf{B}(\mathbf{r})$, whose vector potential $\mathbf{A}(\mathbf{r})$ enters the Hamiltonian via the usual minimal substitution in the kinetic energy

$$
\frac{\hat{p}^{2}}{2 m} \longrightarrow \frac{1}{2 m}\left(\hat{p}-\frac{q}{c} \mathbf{A}(\mathbf{r})\right)^{2} .
$$

A second way in which orbital magnetism can appear is due to currentcurrent interactions, which are part of the Breit interaction $[8,9]$ and therefore 
a relativistic effect. The nonretarded part of this interaction is

$$
-\frac{q^{2}}{c^{2}} \int d^{3} r \int d^{3} r^{\prime} \frac{\mathbf{j}_{p}(\mathbf{r}) \cdot \mathbf{j}_{p}\left(\mathbf{r}^{\prime}\right)}{\left|\mathbf{r}-\mathbf{r}^{\prime}\right|}=-\frac{q}{c} \int d^{3} r \mathbf{j}_{p}(\mathbf{r}) \cdot \mathbf{A}_{H}(\mathbf{r}),
$$

which describes the Hartree-like coupling of currents to the self-induced vector potential, corresponding to the Amperian currents of classical electrodynamics.

Third, in the presence of spin-orbit coupling a nonzero spin magnetization can induce an orbital magnetization. Orbital magnetic moments induced by spin-orbit coupling can be treated within relativistic DFT, and are often described within SDFT by adding a spin-orbit coupling term to the Hamiltonian. Such magnetic moments become important, e.g., in magnetic solids, where spin-orbit coupling produces phenomena such as magneto-crystalline anisotropy $[10,11]$ or magneto-optical effects such as dichroism [12,13].

Finally, and most intriguingly, orbital magnetism can also occur spontaneously in a system with pure Coulomb interactions, if the system minimizes its energy in a current-carrying state $[2,14]$. The resulting currents are in principle functionals of the charge density, because the original formulation of the Hohenberg-Kohn theorem applies in the absence of magnetic fields, but (S)DFT provides no explicit prescription how to calculate the spontaneous orbital currents and their effect on observables. This situation has changed with the advent of nonrelativistic current-density-functional theory, developed by Vignale and Rasolt $[1,2]$, which describes spontaneous currents by introducing in the KohnSham equations a self consistent exchange-correlation $(x c)$ vector potential $\mathbf{A}_{x c}$, which can be nonzero also in the absence of external magnetic fields and of relativistic effects.

In addition to possibly appearing spontaneously, $\mathbf{A}_{x c}$ also becomes nonzero as soon as currents are induced by one of the other three mechanisms. In this case, it constitutes a correction to the external or internal vector potentials, or spin-orbit terms, already present in the Hamiltonian.

CDFT has been applied to the calculation of the effects of orbital magnetism in atoms [15-17], quantum dots [18-20], molecules [21,22] and solids [14,23-25]. In these applications it has become clear that the main challenge of CDFT at the present stage of its development is the construction of reliable and computationally viable approximations to the exchange-correlation vector potential $\mathbf{A}_{x c}$. The present paper explores the possibility to develop such approximations by drawing on analogies to independently developed phenomenological approaches to orbital magnetism.

\section{Phenomenological and first-principles treat- ment of orbital magnetism in density-functional theory}

This section collects, without proofs (which can be found in the original literature), the key equations of both the first-principles (CDFT) and the phe- 
nomenological (OP) approach to orbital magnetism in DFT. As our aim is to unravel a connection between the two, we limit ourselves to providing a brief overview, highlighting the aspects that will allow us to identify the phenomenological approach as a well-defined approximation to the first-principles one.

\subsection{Current-density-functional theory}

To briefly describe the formalism of nonrelativistic CDFT, we first recall the form of the traditional Kohn-Sham (KS) equation of DFT,

$$
\left[-\frac{\hbar^{2}}{2 m} \nabla^{2}+v_{s}(\mathbf{r})\right] \phi_{k}(\mathbf{r})=\epsilon_{k} \phi_{k}(\mathbf{r}) \text {. }
$$

Here the effective single-particle potential, $v_{s}(\mathbf{r})$, is defined as

$$
v_{s}(\mathbf{r})=v(\mathbf{r})+v_{H}(\mathbf{r})+v_{x c}(\mathbf{r}),
$$

where $v(\mathbf{r})$ is the external potential, $v_{H}(\mathbf{r})$ the Hartree electrostatic potential, and $v_{x c}(\mathbf{r})$ the exchange-correlation potential, in which the entire complexity of the many-body problem is hidden [26,27].

By construction, the single-particle orbitals $\phi_{k}$ solving the eigenvalue problem (3) reproduce the density of the interacting system via

$$
n(\mathbf{r})=\sum_{k} \phi_{k}^{*}(\mathbf{r}) \phi_{k}(\mathbf{r}) \text {. }
$$

On the other hand, the paramagnetic current density,

$$
\mathbf{j}_{p}^{K S}(\mathbf{r})=\frac{\hbar}{2 m i} \sum_{k}\left[\phi_{k}^{*}(\mathbf{r}) \nabla \phi_{k}(\mathbf{r})-\phi_{k}(\mathbf{r}) \nabla \phi_{k}^{*}(\mathbf{r})\right],
$$

and the orbital magnetic moment

$$
\left\langle\hat{L}_{z}\right\rangle^{K S}=\frac{m}{\hbar} \int d^{3} r(\hat{\mathbf{z}} \times \mathbf{r}) \cdot \mathbf{j}_{p}^{K S}(\mathbf{r})
$$

following from these orbitals, are, a priori, not guaranteed to have any relation with the true current density and magnetic moment of the interacting system.

The corresponding equations of CDFT have a slightly more complicated form:

$$
\left[\frac{1}{2 m}\left(\frac{\hbar}{i} \nabla-\frac{q}{c} \mathbf{A}_{s}(\mathbf{r})\right)^{2}+V_{s}^{c}(\mathbf{r})\right] \psi_{k}(\mathbf{r})=\epsilon_{k}^{c} \psi_{k}(\mathbf{r}),
$$

where an upper index ' $c$ ' denotes CDFT,

$$
\begin{gathered}
V_{s}^{c}(\mathbf{r})=v_{s}^{c}(\mathbf{r})+\frac{q^{2}}{2 m c^{2}}\left(\mathbf{A}(\mathbf{r})^{2}-\mathbf{A}_{s}(\mathbf{r})^{2}\right), \\
v_{s}^{c}(\mathbf{r})=v(\mathbf{r})+v_{H}(\mathbf{r})+v_{x c}^{c}(\mathbf{r})
\end{gathered}
$$


and

$$
\mathbf{A}_{s}(\mathbf{r})=\mathbf{A}(\mathbf{r})+\mathbf{A}_{x c}(\mathbf{r}) .
$$

Here $v_{x c}^{c}$ and $\mathbf{A}_{x c}$ are the exchange-correlation scalar and vector potentials of CDFT, respectively $[1,2] . \mathbf{A}_{x c}$, in particular, is a gauge invariant functional of the densities $n(\mathbf{r})$ and $\mathbf{j}_{p}(\mathbf{r})$, written $\mathbf{A}_{x c}\left[n, \mathbf{j}_{p}\right](\mathbf{r})$. By setting $\mathbf{A}_{x c} \equiv 0$, one recovers from CDFT the equations of (S)DFT in an external vector potential. The novel feature of CDFT is that $\mathbf{A}_{x c}$ accounts for the orbital degrees-offreedom in the single-particle equations, even in the absence of external fields, and allows calculation of orbital currents directly from a set of Kohn-Sham-type equations.

The single-particle orbitals $\psi_{k}$ solving the more complicated eigenvalue problem (8) reproduce by construction both the density

$$
n(\mathbf{r})=\sum_{k} \psi_{k}^{*}(\mathbf{r}) \psi_{k}(\mathbf{r}),
$$

and the paramagnetic current density

$$
\mathbf{j}_{p}(\mathbf{r})=\frac{\hbar}{2 m i} \sum_{k}\left[\psi_{k}^{*}(\mathbf{r}) \nabla \psi_{k}(\mathbf{r})-\left(\nabla \psi_{k}^{*}(\mathbf{r})\right) \psi_{k}(\mathbf{r})\right]
$$

of the interacting many-body system. The correct orbital magnetic moment can then be obtained from

$$
\left\langle\hat{L}_{z}\right\rangle=\frac{m}{\hbar} \int d^{3} r(\hat{\mathbf{z}} \times \mathbf{r}) \cdot \mathbf{j}_{p}(\mathbf{r}) .
$$

The paramagnetic current alone is not gauge invariant, but the gauge invariant orbital current, $\mathbf{j}_{\text {orb }}(\mathbf{r})$, is simply obtained from

$$
\mathbf{j}_{\text {orb }}(\mathbf{r})=\mathbf{j}_{p}(\mathbf{r})-\frac{q}{m c} n(\mathbf{r}) \mathbf{A}(\mathbf{r}) .
$$

An important property of the effective potential of DFT, $v_{s}(\mathbf{r})$, which is not shared by the CDFT potentials $V_{s}^{c}(\mathbf{r})$ and $\mathbf{A}_{s}(\mathbf{r})$ [28], is its uniqueness: for any given ground-state density $n(\mathbf{r})$ there is, up to an irrelevant additive constant, at most one such local multiplicative potential [26].

Any CDFT calculation requires an approximation for the current dependence of the $E_{x c}$ functional. For the homogeneous three-dimensional electron liquid in strong uniform magnetic fields, the exchange energy is known exactly [29], and the correlation energy has been calculated within the random-phase approximation [30] and the self-consistent local-field corrected Singwi-Tosi-Land-Sjolander scheme [31]. In weak fields, where linear-response theory applies, the exchangecorrelation energy can be expressed in terms of the magnetic susceptibility, for which many-body calculations are available from [32]. The exchange-correlation energy of two-dimensional electron liquids in uniform magnetic fields has been much studied in the context of the fractional Quantum Hall effect in quasi-twodimensional semiconductor heterostructures [33]. 


\subsection{Orbital-polarization terms}

In an independent take on the problem of orbital magnetism, originating with Brooks and collaborators $[3,4]$, phenomenological orbital-polarization terms are introduced in the formal framework of SDFT. This effort is motivated by observing that SDFT does account for Hund's first rule (dealing with spin angular momentum) and can be extended to account for Hund's third rule (due to spinorbit coupling), but does not contain an obvious ingredient corresponding to Hund's second rule (dealing with orbital angular momentum).

In the absence of external magnetic fields, the exact exchange-correlation functional of SDFT doubtlessly would predict the correct orbital magnetic moments and orbital currents, as functionals of the spin and charge densities, but these functionals are not known. In practice, such quantities are therefore often calculated directly from the orbitals of SDFT. We note that there are two separate issues here: One is the use of SDFT orbitals to calculate orbital magnetic moments, although these orbitals are constructed to reproduce only the charge and spin densities, not the orbital currents. The other is the use of an approximate SDFT functional (the LSDA) in these calculations.

In practice, the resulting orbital magnetic moments strongly underestimate experimental values for transition metals [34], and make wrong predictions for, e.g., the volume collapse in lanthanides [4] and the band gap of transition-metal oxides $[5,35]$. This behaviour was interpreted as a consequence of the inapplicability of Hund's second rule to the electron gas, on which the LSDA is based. To account for Hund's second rule, phenomenological orbital angular momentum dependent corrections to the LSDA were proposed by drawing on analogies with angular momentum dependent terms in the multiplet splitting of atoms $[3,4]$. Significant improvement of magnetic moments and related quantities is obtained from this so-called orbital-polarization approach [3-5, 10,11, 23, 34, 36-39].

In Sec. 4, we explain that this empirical success can be understood if the OP term is not considered a better SDFT functional than the LSDA, but rather an approximate CDFT functional. As such, it produces orbitals that can reproduce charge, spin and current densities, and thus also orbital magnetic moments.

The original proposal for systems with open shells of $4 f$ electrons was to add the OP term (today also known as Brooks term)

$$
\Delta E^{B}=-\frac{1}{2} E^{3} L^{2}
$$

to the total-energy functional of SDFT. Here $E^{3}$ is a Racah parameter defined in terms of Slater integrals $F_{k}$ as $E^{3}=\left(5 F_{2}+6 F_{4}-91 F_{6}\right) / 3$ [40,41], and $L$ is the total orbital angular momentum of the open-shell ion defined as $[4,5,10]$

$$
L:=\sum_{i}\left\langle\hat{l}_{z}\right\rangle=\left\langle\hat{L}_{z}\right\rangle=\sum_{n l m_{l} \sigma} \gamma_{n l m_{l} \sigma} m_{l}
$$

in terms of a sum over occupation numbers $\gamma_{n l m_{l} \sigma}$ of single-particle orbitals. Expressions (16) and (17) are motivated by noting the presence of similar 
terms in the vector model of the multiplet splitting of atoms with open $f$ shells $[4-6,40,41]$, but their inclusion in an SDFT calculation is entirely ad hoc. Nevertheless, by adopting Eq. (16) as a starting point, simple and empirically successful correction terms to the single-particle equations of SDFT are obtained [3-5, 10, 11, 23, 34, 36-39].

The original OP idea was subsequently extended to $d$-electrons, for which Norman proposed [5]

$$
\Delta E^{N d^{\prime}}=-\frac{1}{2} B L^{2}
$$

where $B$ is the Racah parameter $B=F_{2}-5 F_{4}[40,41]$.

Further refinements are based on a more complete treatment of atomic multiplet splitting [6,7] and crystal-field effects [35]. For $d$-electrons, Norman [6] proposed the expression

$$
\Delta E^{N d}=2.25 n_{d}\left(5-n_{d}\right) B-1.5 L(L+1) B
$$

where $n_{d}$ is the occupation number of the $3 d$ orbitals, and for $f$-electrons,

$$
\Delta E^{N f}=\left(-2 n_{f}^{2}+14 n_{f}\right) E^{3}-L(L+1) E^{3},
$$

where $n_{f}$ is the occupation number of the $4 f$ orbitals. Shick e Gubanov [7] refined Norman's argument for $f$-electrons, and obtained

$$
\Delta E^{S G}=-\frac{3}{2}\left[L(L+1)-6 g\left(G_{2}\right)\right] E^{3},
$$

where $g\left(G_{2}\right)=\left[6 n_{f}\left(7-n_{f}\right)+41 n_{f}^{2}\left(7-n_{f}\right)^{2}-2 n_{f}^{3}\left(7-n_{f}\right)^{3}\right] /\left(2^{2} 3^{3} 5\right)$. This expression by construction recovers the correct splitting of the highest-spin multiplet of the isolated ion $[6,7]$.

Although expressions (19), (20), and (21) give a better description of atomic multiplets than Eqs. (16) and (18), applications to solids containing transitionmetal and rare-earth atoms $[4,5,10,11,23,34,36,37,39]$ mostly employed the simpler expressions (16) and (18), which already lead to substantial improvement of orbital magnetic moments and related quantities, as compared to uncorrected LSDA. In Sec. 4, we therefore mainly focus on the simple expressions (16) and (18).

We note in passing that apparently no OP term has been proposed for $p$ electrons, although the physical motivation and the mathematical argument leading to the OP terms for $d$ and $f$ electrons would apply to them, too. From an analysis of the multiplet splitting of the $p$ configurations [41], we find that the $p$-electron OP term should have the form

$$
\Delta E^{p}=\frac{3}{2}\left[n_{p}\left(6-n_{p}\right)-L(L+1)-4 S(S+1)\right] F_{2},
$$

which is obtained by subtracting from the energy of the terms arising from the $p$-shell their spherical average. This expression for $p$ electrons is valid for all $S$, in contrast to expressions (19) for $d[6]$ and (20) and (21) for $f[6,7]$ electrons, 
which hold only for the highest spin multiplet, where $S=n_{d} / 2$ and $S=n_{f} / 2$, respectively. In analogy to Eqs. (16) and (18), a simpler version, expected to simulate the effect of (22), is

$$
\Delta E^{p^{\prime}}=-\frac{1}{2} F_{2} L^{2} .
$$

Finally, we remark that $d$ and $f$-electron OP terms of similar form to (19), (20) and (21) have been obtained also within relativistic DFT [38]. In that work, as well as in Refs. [34,39], it was pointed out that there should be a connection between the various phenomenological orbital-polarization corrections and the first-principles formalism of (relativistic or nonrelativistic) CDFT. In the next section we demonstrate this connection.

\section{Connection between the phenomenological and the first-principles approach}

In this section we establish a connection between the OP terms and CDFT. This connection is obtained in two different ways, once by analysing single-particle Hamiltonians (Sec. 4.1), and once by considering total energies (Sec. 4.2). The method of analysis is different in both cases, but the final results are the same.

\subsection{Single-particle energies}

In the absence of external magnetic fields, the Kohn-Sham single-particle Hamiltonians of CDFT and DFT take the form

$$
\hat{H}^{c}=-\frac{\hbar^{2}}{2 m} \nabla^{2}-\frac{i \hbar e}{2 m c}\left[\mathbf{A}_{x c}(\mathbf{r}) \cdot \nabla+\nabla \cdot \mathbf{A}_{x c}(\mathbf{r})\right]+v(\mathbf{r})+v_{H}(\mathbf{r})+v_{x c}^{c}(\mathbf{r})
$$

and

$$
\hat{H}=-\frac{\hbar^{2}}{2 m} \nabla^{2}+v(\mathbf{r})+v_{H}(\mathbf{r})+v_{x c}(\mathbf{r}),
$$

respectively. There difference is thus given by the operator

$$
\Delta \hat{H}=\hat{H}^{c}-\hat{H}=-\frac{i \hbar e}{2 m c}\left[\mathbf{A}_{x c}(\mathbf{r}) \cdot \nabla+\nabla \cdot \mathbf{A}_{x c}(\mathbf{r})\right]+\Delta v_{x c}(\mathbf{r}),
$$

where $\Delta v_{x c}(\mathbf{r})=v_{x c}^{c}(\mathbf{r})-v_{x c}(\mathbf{r})$. A perturbative treatment of the first term of this difference was suggested in Ref. [42] and applied mainly to $p$ electrons in $[15,16]$. In the context of magnetism, however, our main interest is in $d$ and $f$ electrons, for which the difference between both Hamiltonians may be too large to justify a low-order perturbation treatment. Moreover, the functional used in $[15,16]$, as well as in many other applications of CDFT [21-23], is based on the electron liquid, which at stronger magnetic fields displays quantum oscillations [30,31], which are incorrect for finite atomic and molecular systems. 
Independently of the size of the various terms, the identity $\nabla \cdot\left[n(\mathbf{r}) \mathbf{A}_{x c}(\mathbf{r})\right]=$ 0 always holds $[1,2]$, and can be used to write $\Delta \hat{H}$ in spherical polar coordinates $(r, \theta, \varphi)$ as

$$
\begin{array}{r}
\Delta \hat{H}=\Delta v_{x c}(\mathbf{r})- \\
2 m c\left[2\left(A_{x c}^{r}(\mathbf{r}) \frac{\partial}{\partial r}+\frac{1}{r} A_{x c}^{\theta}(\mathbf{r}) \frac{\partial}{\partial \theta}+\frac{1}{r \sin \theta} A_{x c}^{\varphi}(\mathbf{r}) \frac{\partial}{\partial \varphi}\right)-\frac{\nabla n(\mathbf{r})}{n(\mathbf{r})} \cdot \mathbf{A}_{x c}(\mathbf{r})\right] .
\end{array}
$$

This is the complete set of (nonrelativistic) corrections to DFT predicted by CDFT in the absence of external fields.

We can now compare this to the various ad hoc corrections listed in Sec. 3.2 According to Janak's theorem [43], the DFT Kohn-Sham eigenvalue is obtained from the total energy by differentiating with respect to the occupation number,

$$
\epsilon_{n l m_{l} \sigma}=\frac{\partial E}{\partial \gamma_{n l m_{l} \sigma}} .
$$

If this relation, which also holds in SDFT and CDFT, is applied to the SDFT functional augmented by the orbital polarization term (16), and the Racah parameter is kept fixed during the differentiation, it follows that the ad hoc OP correction to the total energy corresponds to a correction to the single-particle energies of the form

$$
\Delta \epsilon^{B}=-E^{3} L m_{l}
$$

which can in turn can be interpreted as a result of the operator

$$
\Delta \hat{H}^{B}=-E^{3} L \hat{l}_{z}
$$

added to the single-particle equation of SDFT. Similarly,

$$
\begin{gathered}
\Delta \hat{H}^{N d^{\prime}}=-B L \hat{l}_{z}, \\
\Delta \hat{H}^{p^{\prime}}=-F_{2} L \hat{l}_{z} \\
\Delta \hat{H}^{N d}=2.25\left(5-2 n_{d}\right) B-3 B\left(L+\frac{1}{2}\right) \hat{l}_{z}, \\
\Delta \hat{H}^{N f}=\left(-4 n_{f}+14\right) E^{3}-2 E^{3}\left(L+\frac{1}{2}\right) \hat{l}_{z},
\end{gathered}
$$

and

$$
\begin{array}{r}
\Delta \hat{H}^{S G}=-3 E^{3}\left(L+\frac{1}{2}\right) \hat{l}_{z}+\frac{9}{2^{2} 3^{3} 5} \\
{\left[\left(7-n_{f}\right)\left(6-82 n_{f}^{2}\right)+\left(7-n_{f}\right)^{2}\left(82 n_{f}+6 n_{f}^{3}\right)-6 n_{f}^{2}\left(7-n_{f}\right)^{3}-6 n_{f}\right] E^{3} .}
\end{array}
$$

We note that if the Racah term is not treated as a number, but as a matrix element involving single-particle radial orbitals [40,41], additional terms appear on the right-hand side of these equations. (We return to this issue at the end of Sec. 4.2, from a slightly different point of view.) 
Since $\hat{l}_{z}=-i \partial / \partial \varphi$, the single-particle operators resulting from the OP terms, Eqs. (30), (31) and (32), can be cast in the form of Eq. (27), with

$$
\mathbf{A}_{x c}^{O P}(\mathbf{r})=-\frac{m c}{\hbar e} R_{l} r L \sin \theta \hat{\varphi},
$$

along with

$$
v_{x c}^{c, O P}(\mathbf{r})=v_{x c}(\mathbf{r}),
$$

and $A_{x c}^{O P, r}(\mathbf{r})=A_{x c}^{O P, \theta}(\mathbf{r})=\nabla n(\mathbf{r}) \cdot \mathbf{A}_{x c}^{O P}(\mathbf{r})=0$. In these equations $\hat{\varphi}$ is the unit vector in the azimuthal direction, and $R_{l}=E^{3}, B, F_{2}$, for $f, d$, and $p$ electrons, respectively.

This way of writing the OP correction shows that it enters the KS Hamiltonian in the same way as the $x c$ vector potential of CDFT. To complete the proof that it really is such a potential, we need to write it as a functional of the current density, $\mathbf{A}_{x c}^{O P}\left[\mathbf{j}_{p}(\mathbf{r})\right]$, which is easily accomplished by means of Eqs. (14) and (17).

We note that $v_{x c}^{c, O P}(\mathbf{r})=v_{x c}(\mathbf{r})$ implies $\Delta v_{x c}^{O P}(\mathbf{r})=0$, which may be interpreted as a weak coupling approximation, in which the self-consistent effect of currents on the electric potential is considered small. The neglect of the radial and polar components of $\mathbf{A}_{x c}(\mathbf{r})$ is correct for systems with spherical symmetry, where $\nabla n(\mathbf{r}) \cdot \mathbf{A}_{x c}$ also vanishes. The orbital-polarization terms can thus be identified as weak-coupling, spherically symmetric approximations to the selfconsistent $x c$ vector potential of CDFT.

This conclusion was anticipated in Refs. [34,39], where it was, however, argued that the physics described by the Brooks term was distinct from that of CDFT. Our present point of view is different: CDFT is a general framework for describing all nonrelativistic effects of orbital magnetism, and to the extent that the orbital polarization terms are justifiable, they must be specific approximations to the general framework of CDFT.

The other orbital-polarization terms can also be identified as approximations to $\mathbf{A}_{x c}$. Eqs. (33) and (34) lead to

$$
\mathbf{A}_{x c}^{N d}(\mathbf{r})=-3 \frac{m c}{\hbar e} B\left(L+\frac{1}{2}\right) r \sin \theta \hat{\varphi}
$$

and

$$
v_{x c}^{c, N d}(\mathbf{r})=v_{x c}(\mathbf{r})+2.25\left(5-2 n_{d}\right) B
$$

for $d$ electrons, and

$$
\mathbf{A}_{x c}^{N f}(\mathbf{r})=-2 \frac{m c}{\hbar e} E^{3}\left(L+\frac{1}{2}\right) r \sin \theta \hat{\varphi}
$$

and

$$
v_{x c}^{c, N f}(\mathbf{r})=v_{x c}(\mathbf{r})+\left(-4 n_{f}+14\right) E^{3}
$$

for $f$ electrons. Interestingly, the scalar $x c$ potentials are also modified by these proposals. However, the modification is a constant shift, independent of position, whereas $\Delta v_{x c}(\mathbf{r})=v_{x c}^{c}(\mathbf{r})-v_{x c}(\mathbf{r})$ should, generically, depend on $\mathbf{r}$. 
Finally, the proposal of Shick e Gubanov implies

$$
\mathbf{A}_{x c}^{S G}(\mathbf{r})=-3 \frac{m c}{\hbar e} E^{3}\left(L+\frac{1}{2}\right) r \sin \theta \hat{\varphi}
$$

and

$$
v_{x c}^{c, S G}(\mathbf{r})=v_{x c}(\mathbf{r})+\frac{E^{3}}{30}\left[21+2003 n_{f}-1890 n_{f}^{2}+670 n_{f}^{3}-105 n_{f}^{4}+6 n_{f}^{5}\right] .
$$

All these expressions for $\mathbf{A}_{x c}(\mathbf{r})$ and $v_{x c}^{c}(\mathbf{r})$ can be implemented self-consistently, by recalculating the Racah parameters and the occupation numbers at every iteration, although such a self-consistent recalculation is not consistent with treating these parameters as numbers when deducing the eigenvalue correction from the total-energy correction.

\subsection{Total energies}

The connection between the CDFT $x c$ potentials and the orbital polarization corrections, summarized in Eqs. (36) and (37) was obtained by comparing operators in the single-particle equations of CDFT and of the SDFT + OP approach. The operator in the SDFT + OP equations was obtained by means of Janak's theorem, from partial differentiation of the total energy with respect to occupation numbers.

Alternatively, one can follow the established DFT way to obtain potentials directly from total energies, by variational differentiation with respect to densities, according to $[1,2]$

$$
v_{x c}^{c}(\mathbf{r})=\frac{\delta E_{x c}^{c}\left[n, \mathbf{j}_{p}\right]}{\delta n(\mathbf{r})}
$$

and

$$
\frac{e}{c} \mathbf{A}_{x c}(\mathbf{r})=\frac{\delta E_{x c}^{c}\left[n, \mathbf{j}_{p}\right]}{\delta \mathbf{j}_{p}(\mathbf{r})}
$$

Application of these definitions to the DFT total energy expression, augmented by the OP terms (16), (18) or (23), leads to

$$
\begin{aligned}
v_{x c}^{c, O P}(\mathbf{r}) & =\frac{\delta E_{x c}[n]}{\delta n(\mathbf{r})}-\frac{1}{2} \frac{\delta}{\delta n(\mathbf{r})}\left[R_{l}\left\langle\hat{L}_{z}\right\rangle^{2}\right] \\
& =v_{x c}(\mathbf{r})-\frac{1}{2} \frac{\delta}{\delta n(\mathbf{r})}\left[R_{l}\left\langle\hat{L}_{z}\right\rangle^{2}\right]
\end{aligned}
$$

and

$$
\begin{aligned}
\frac{e}{c} \mathbf{A}_{x c}^{O P}(\mathbf{r}) & =\frac{\delta E_{x c}[n]}{\delta \mathbf{j}_{p}(\mathbf{r})}-\frac{1}{2} \frac{\delta}{\delta \mathbf{j}_{p}(\mathbf{r})}\left[R_{l}\left\langle\hat{L}_{z}\right\rangle^{2}\right] \\
& =0-R_{l}\left\langle\hat{L}_{z}\right\rangle \frac{\delta}{\delta \mathbf{j}_{p}(\mathbf{r})}\left\langle\hat{L}_{z}\right\rangle-\frac{1}{2}\left\langle\hat{L}_{z}\right\rangle^{2} \frac{\delta R_{l}}{\delta \mathbf{j}_{p}(\mathbf{r})}
\end{aligned}
$$


These equations can be simplified by using Eq. (14) to calculate $\delta\left\langle\hat{L}_{z}\right\rangle / \delta \mathbf{j}_{p}(\mathbf{r})$. Since within $\operatorname{CDFT} n(\mathbf{r})$ and $\mathbf{j}_{p}(\mathbf{r})$ are independent variables, we arrive at

$$
v_{x c}^{c, O P}(\mathbf{r})=v_{x c}(\mathbf{r})-\frac{1}{2}\left\langle\hat{L}_{z}\right\rangle^{2} \frac{\delta R_{l}}{\delta n(\mathbf{r})}
$$

and

$$
\mathbf{A}_{x c}^{O P}(\mathbf{r})=-\frac{m c}{\hbar e} R_{l} r \sin \theta\left\langle\hat{L}_{z}\right\rangle \hat{\varphi}-\frac{c}{2 e}\left\langle\hat{L}_{z}\right\rangle^{2} \frac{\delta R_{l}}{\delta \mathbf{j}_{p}(\mathbf{r})} .
$$

If we again treat the Racah parameters as numbers, and not as self-consistent functionals of the densities, we finally obtain

$$
v_{x c}^{c, O P}(\mathbf{r})=v_{x c}(\mathbf{r})
$$

and

$$
\mathbf{A}_{x c}^{O P}(\mathbf{r})=-\frac{m c}{\hbar e} R_{l} r \sin \theta\left\langle\hat{L}_{z}\right\rangle \hat{\varphi} .
$$

These are the same relations obtained in the preceding section from Janak's theorem and partial differentiation with respect to occupation numbers. Just as there, the Racah parameters must be treated as fixed numbers in order to arrive at the standard form of the OP term. We note that if the Racah coefficients are differentiated consistently also in the derivation via Janak's theorem, one obtains, by application of the chain rule in the form

$$
\frac{\partial R_{l}}{\partial \gamma}=\int d^{3} r\left[\frac{\delta R_{l}}{\delta n(\mathbf{r})} \frac{\partial n(\mathbf{r})}{\partial \gamma}+\frac{\delta R_{l}}{\delta \mathbf{j}_{p}(\mathbf{r})} \frac{\partial \mathbf{j}_{p}(\mathbf{r})}{\partial \gamma}\right],
$$

the same extra terms appearing on the right-hand side of Eqs. (48) and (49). To the best of our knowledge, consequences of these additional $\left\langle L_{z}\right\rangle^{2}$ term have never been systematically explored.

\section{Conclusions}

The $f$-electron orbital polarization term proposed by Brooks at al. [3,4], its generalization to $d$ electrons [5], and their refinements and generalizations $[6,7,35]$, have been identified as specific approximations to $\mathbf{A}_{x c}(\mathbf{r})$ and $v_{x c}^{c}(\mathbf{r})$ of CDFT. This identification provides formal justification for their introduction into the Kohn-Sham Hamiltonian of (S)DFT: by using an orbital polarization correction one actually simulates certain aspects of CDFT within the computational framework of (S)DFT.

The existence of such a connection implies that the OP terms should not be interpreted just as corrections to the LSDA of SDFT, although this may have been their original motivation, but also (and perhaps principally!) as a step from SDFT to CDFT. Hence, from the present point of view, the OP terms address both issues facing the calculation of orbital moments in SDFT that were mentioned at the beginning of Sec. 3.2 correction of electron-gas-based $x c$ functionals for Hund's second rule, and substitution of the SDFT orbitals 
by CDFT orbitals that reproduce the orbital currents. We stress that this also holds for the $p$-electron OP terms (22) and (23), proposed in this work.

Explicit expressions for $\mathbf{A}_{x c}$ and $v_{x c}^{c}(\mathbf{r})$ that can be used in CDFT are scarce, and mostly based on many-body calculations of the energy of the uniform electron gas in certain ranges of external magnetic fields [2,29-33]. Explicit, albeit approximate, expressions for $\mathbf{A}_{x c}$ and $v_{x c}^{c}(\mathbf{r})$ that by construction go beyond local-density approximations may be useful in applications to finite systems, and constitute starting points for the development of more refined functionals.

As an example, by combining (51) with (14) we obtain the explicit currentdensity functional

$$
\mathbf{A}_{x c}^{O P}\left[\mathbf{j}_{p}\right](\mathbf{r})=-\frac{m^{2} c}{\hbar^{2} e} R_{l} r \sin \theta\left[\int d^{3} r r \sin \theta j_{p}^{\varphi}(\mathbf{r})\right] \hat{\varphi} .
$$

The empirical success of the OP concept in solid-state physics suggests that this may be a useful and reliable expression for $\mathbf{A}_{x c}$ of molecular and solid sys-

tems containing open-shell atoms. As this expression is nonperturbative, and not based on the electron liquid, it is expected to be more appropriate for systems containing open-shell atoms than the linear-response LDA $[2,15,16,21-23]$. This expression also does not suffer from quantum oscillations that stem from the electron liquid but are incorrect for the atomic and molecular systems $[30,31]$. It may therefore constitute a useful starting point also for applications of CDFT to molecular systems.

Acknowledgments This work was supported by FAPESP and CNPq. KC thanks Angela Klautau and Helmut Eschrig for useful conversations about orbital polarization terms.

\section{References}

[1] G. Vignale and M. Rasolt, Phys. Rev. Lett. 59, 2360 (1987).

[2] G. Vignale and M. Rasolt, Phys. Rev. B 37, 10685 (1988).

[3] M. S. S. Brooks, Physica 130B, 6 (1985).

[4] O. Eriksson, M. S. S. Brooks and B. Johansson, Phys. Rev. B 41, 7311 (1990).

[5] M. R. Norman, Phys. Rev. Lett. 64, 1162 (1990).

[6] M. R. Norman, Int. J. Quantum Chem. S25, 431 (1991).

[7] A. B. Shick and V. A. Gubanov, Phys. Rev. B 49, 12860 (1994).

[8] P. Strange, Relativistic Quantum Mechanics with Applications in Condensed Matter and Atomic Physics (Cambridge University Press, Cambridge, 1998). 
[9] Pyykkö, P. Adv. in Quantum Chem., 1978, Vol. 11, p. 353.

[10] O. Eriksson and J. Wills, First Principles Theory of Magneto-Crystalline Anisotropy, in Springer Lecture Notes in Physics, Vol. 535, p. 247 (1999).

[11] O. Eriksson, First Principle Theory of Magnetism for Materials with Reduced Dimensionality, in Springer Lecture Notes in Physics, Vol. 580, p. 243 (2001).

[12] H. Ebert, Rep. Prog. Phys. 59, 1665 (1996).

[13] K. Capelle, E. K. U. Gross and B. L. Györffy, Phys. Rev. Lett. 78, 3753 (1997).

[14] M. Rasolt and F. Perrot, Phys. Rev Lett. 69, 2563 (1992).

[15] E. Orestes, T. Marcasso and K. Capelle, Phys. Rev. A 68, 022105 (2003).

[16] E. Orestes, A. B. F. da Silva and K. Capelle, Int. J. Quantum Chem. 103, 516 (2005).

[17] J. Tao and J. P. Perdew, Phys. Rev. Lett. 95, 196403 (2005).

[18] M. Ferconi and G. Vignale, Phys. Rev. B 50, 14722 (1994).

[19] O. Steffens, U. Rössler and M. Suhrke, Europhys. Lett. 42, 529 (1998).

[20] M. Pi, M. Barranco, A. Emperador, E. Lipparini and Ll. Serra, Phys. Rev. B 57, 14783 (1998).

[21] S. M. Colwell and N. C. Handy, Chem. Phys. Lett. 217, 271 (1994).

[22] A. M. Lee, S. M. Colwell and N. C. Handy, Chem. Phys. Lett. 229, 225 (1994).

[23] H. Ebert, M. Battocletti and E. K. U. Gross, Europhys. Lett. 40, 545 (1997).

[24] G. Vignale, Phys. Rev. B 47, 10105 (1993).

[25] S. Sharma, S. Pittalis, S. Kurth, S. Shallcross, J. K. Dewhurst and E. K. U. Gross, Phys. Rev. B 76, 100401 (2007).

[26] R. M. Dreizler and E. K. U. Gross, Density Functional Theory (Springer, Berlin, 1990).

[27] R. G. Parr and W. Yang, Density-Functional Theory of Atoms and Molecules (Oxford University Press, Oxford, 1989).

[28] K. Capelle and G. Vignale, Phys. Rev. B 65, 113106 (2002).

[29] R. W. Danz and M. L. Glasser, Phys. Rev. B 4, 94 (1971). 
[30] P. Skudlarski and G. Vignale, Phys. Rev. B 48, 8547 (1993).

[31] Y. Takada and H. Goto, J. Phys.: Condens. Matter 10, 11315 (1998).

[32] G. Vignale, M. Rasolt and D. J. W. Geldart, Phys. Rev. B 37, 2502 (1988).

[33] A. Wensauer and U. Rössler, Phys. Rev. B 69, 155302 (2004).

[34] T. Huhne, C. Zecha, H. Ebert, P. H. Dederichs and R. Zeller, Phys. Rev. B 58, 10236 (1998).

[35] M. R. Norman, Phy. Rev. B 44, 1364 (1991).

[36] A. B. Klautau and S. Frota-Pessoa, Phys. Rev. B 70, 193407 (2004).

[37] S. Frota-Pessoa, Phys. Rev. B 69, 104401 (2004).

[38] H. Eschrig, M. Sargolzaei, K. Koepernik and M. Richter, Europhys. Lett. 72, $611(2005)$.

[39] H. Ebert and M. Battocletti, Solid State Commun. 98, 785 (1996).

[40] G. Racah, Phys. Rev. 62, 438 (1942).

[41] J. S. Griffith, The Theory of Transition-Metal Ions (Cambridge University Press, Cambridge, 1961).

[42] K. Capelle, Phys. Rev. A 60, 733 (1999).

[43] J. F. Janak, Phys. Rev. B 18, 7165 (1978). 